\title{
Makroökonomische Folgen der demografischen Alterung
}

\author{
Simulationen für Deutschland, Japan und die USA bis 2050
}

\begin{abstract}
Der demografische Wandel ist in den meisten Industrienationen mit einer Alterung und Schrumpfung der Erwerbsbevölkerung verbunden. Daraus ergeben sich erhebliche Konsequenzen für zentrale makroökonomische Größen wie das Bruttoinlandsprodukt, die Arbeitsproduktivität, die Ersparnisse und Investitionen sowie den Leistungsbilanzsaldo. Angesichts des in den nächsten Jahrzehnten zu erwartenden demografischen Wandels müssen vor allem die stark alternden Länder Deutschland und Japan mit einer spürbaren Dämpfung des Wirtschaftswachstums rechnen. Allerdings kann z. B. der technologische Fortschritt in Form von Automatisierung und Digitalisierung diesen Entwicklungen entgegenwirken.
\end{abstract}

\begin{abstract}
Eine umfangreiche Studie, die das Österreichische Institut für Wirtschaftsforschung (WIFO) für die Bertelsmann Stiftung (2019) erarbeitet hat, untersucht, welchen Ein-

(C) Der/die Autor(en) 2020. Open Access: Dieser Artikel wird unter der Creative Commons Namensnennung 4.0 International Lizenz (https:// creativecommons.org/licenses/by/4.0/deed.de) veröffentlicht.

Open Access wird durch die ZBW - Leibniz-Informationszentrum Wirtschaft gefördert.
\end{abstract}

Dr. Thieß Petersen ist Senior Advisor der Bertelsmann Stiftung im Projekt "Global Economic Dynamics" und Lehrbeauftragter an der Europa-Universität Viadrina in Frankfurt/Oder.

Dr. Martina Lizarazo López leitet das Projekt „Demografieresilienz und Teilhabe“ bei der Bertelsmann Stiftung.

Priv.-Doz. Dr. Serguei Kaniovski ist wissenschaftlicher Mitarbeiter im Forschungsbereich „Makroökonomie und europäische Wirtschaftspolitik" des Österreichischen Instituts für Wirtschaftsforschung (WIFO).

Dr. Thomas Url ist wissenschaftlicher Mitarbeiter im Forschungsbereich „Makroökonomie und europäische Wirtschaftspolitik" am WIFO. fluss die zu erwartende demografische Entwicklung in sieben ausgewählten hoch entwickelten Industrieländern (Deutschland, Frankreich, Italien, Österreich, Spanien, USA und Japan) auf zentrale makroökonomische GröBen hat. Die Simulationsrechnungen reichen bis zum Jahr 2050 und beruhen auf einer modifizierten Version der von der EU-Kommission (2017) zur Erstellung des Ageing Report eingesetzten Methode. Die Kommissionsmethode wird dabei um Ergebnisse von Regressionsanalysen ergänzt, welche die Zusammenhänge zwischen einer sich ändernden Altersstruktur der Bevölkerung und zentralen makroökonomischen Größen auf Basis vergangener Entwicklungen seit 1980 empirisch schätzen.

\section{Alterung und gesamtwirtschaftliche Entwicklung}

Von besonderer Bedeutung für den materiellen Wohlstand der Menschen sind letztendlich die Auswirkungen der gesellschaftlichen Alterung auf das reale Bruttoinlandsprodukt (BIP) je Einwohner*in. Dieses hängt wiederum maßgeblich von der Arbeitsproduktivität und dem zahlenmäßigen Verhältnis von Erwerbstätigen zu altersbedingt nicht mehr (oder noch nicht) erwerbstätigen Personen ab.

Im Hinblick auf die individuelle Arbeitsproduktivität eines Menschen und dessen Alter ist ein buckelförmiger Verlauf über die Lebenszeit zu erwarten. Junge Menschen befinden sich entweder noch in der Ausbildung oder am Beginn ihrer Erwerbskarriere. Sie verfügen nur über ein geringes Erfahrungswissen und haben in der Regel eine vergleichsweise geringe Arbeitsproduktivität. Mit steigendem Alter nimmt das Erfahrungswissen zu, sodass auch 
die Produktivität der Arbeitskräfte steigt. Im höheren Alter gehen die physische und die kognitive Leistungsfähigkeit langsam zurück. Anfangs kann dies noch durch Erfahrungsleistungen kompensiert werden, aber mit weiter fortschreitendem Alter ist mit einem Rückgang der Arbeitsproduktivität zu rechnen. Empirische Studien zeigen, „dass die individuelle Arbeitsproduktivität mit zunehmendem Alter zunächst steigt und dann sinkt" (Ademmer et al., 2017). Die höchste Produktivität erreichen Menschen im Durchschnitt mit einem Alter von rund 50 Jahren (Bertelsmann Stiftung, 2019; Skirbek, 2004, 143). Wenn die Alterung der gesamtwirtschaftlich vorhandenen Erwerbstätigen dazu führt, dass große Teile der Beschäftigten dieses Alter überschreiten, hat das einen produktivitätsdämpfenden Effekt für die Volkswirtschaft.

In einer alternden Gesellschaft trifft eine sinkende Zahl von Erwerbspersonen auf eine steigende Zahl von Rentnern. Die gesamtgesellschaftliche Erwerbsquote, definiert als Anteil der Erwerbspersonen an der Gesamtbevölkerung, geht dadurch zurück. Unter ansonsten unveränderten ökonomischen Rahmenbedingungen bedeutet dies einen Rückgang des materiellen Wohlstands je Kopf.

Im Hinblick auf die Ersparnisbildung der privaten Haushalte lassen sich vereinfachend folgende drei Altersgruppen unterscheiden:

- Junge Menschen und junge Erwerbstätige erzielen entweder noch gar kein eigenes Einkommen oder allenfalls geringe Arbeitseinkommen. Ihre Möglichkeiten, Ersparnisse zu bilden, sind daher äußerst gering bzw. nicht existent.

- Erwerbstätige in mittleren und höheren Altersgruppen: Mit zunehmender Berufserfahrung nehmen die Arbeitseinkommen in der Regel zu. Dies erhöht die Möglichkeit, Ersparnisse anzusammeln. Gleichzeitig hat diese Altersgruppe einen hohen Anreiz, durch Ersparnisse Rücklagen für das Alter zu bilden.

- Personen im Rentenalter: Da die Renten und Pensionen niedriger ausfallen als die Arbeitseinkommen, geht die Ersparnisbildung zurück. Gleichzeitig setzen betriebliche und private Rentenzahlungen ein, und das in der Erwerbsphase gebildete Vermögen wird teilweise aufgelöst, um den materiellen Lebensstandard halten zu können. Es kommt also zu einem Entsparen.

Infolgedessen sind zwei grundsätzliche Entwicklungen zu erwarten: In einer alternden Gesellschaft - verstanden als eine Gesellschaft, in der die Zahl der Kinder und Jugendlichen sinkt und die Beschäftigten älter werden, gleichzeitig aber noch verhältnismäßig wenige Menschen im Rentenalter sind - steigt die gesamtwirtschaftliche Sparquote. In einer alten Gesellschaft - verstanden als eine Gesellschaft, in der es viele Menschen im Rentenalter gibt und im Verhältnis dazu die Zahl der Menschen im Erwerbsalter kleiner wird - sinkt die Sparquote.

Ersparnisse entsprechen dem inländischen Kapitalangebot einer Volkswirtschaft, das durch ausländisches Kapitalangebot ergänzt werden kann. Wenn die Ersparnisse wegen der Alterung der Bevölkerung zurückgehen, resultiert aus dem geringeren Kapitalangebot ein steigender Zins. Der Zinsanstieg führt zu geringeren Investitionen. Ein zweiter investitionsdämpfender Effekt der gesellschaftlichen Alterung ergibt sich aus dem Umstand, dass die Bevölkerung im erwerbsfähigen Alter schrumpft. Ceteris paribus sind dann geringere gesamtwirtschaftliche Investitionen erforderlich, um diese Personen mit Maschinen und Arbeitsmitteln auszustatten. Das theoretisch erwartete Resultat ist ein Rückgang der Investitionsquote.

Empirisch sind die Exporte und Importe eines Landes die zentralen Determinanten des Leistungsbilanzsaldos einer Volkswirtschaft. Wichtige Einflussgrößen für die Exporte und Importe eines Landes sind wiederum dessen internationale Wettbewerbsfähigkeit und die Binnennachfrage. Weil sich eine alternde Bevölkerung durch eine nachlassende Arbeitsproduktivität und einen Anstieg des Preisniveaus (weil ein wachsender Anteil der Bevölkerung mehr Güter und Dienstleistungen konsumiert, als er herstellt) auszeichnet, geht die internationale Wettbewerbsfähigkeit der Volkswirtschaft zurück. Deshalb werden weniger Güter und Dienstleitungen exportiert und gleichzeitig tendenziell mehr importiert. Daraus resultiert ein Rückgang des Leistungsbilanzsaldos. Ein hoher Anteil älterer Menschen bewirkt darüber hinaus einen Rückgang der gesamtwirtschaftlichen Sparquote, der Anteil der Konsumnachfrage am BIP nimmt zu. Daher bleiben weniger Güter und Dienstleistungen für den Export übrig. Die Differenz zwischen den Exporten und Importen schrumpft folglich.

\section{Methodische Grundlagen}

Um den Einfluss der bis 2050 zu erwartenden demografischen Alterung auf die makroökonomische Entwicklung der sieben Industrieländer simulieren zu können, wird ein zweistufiges Verfahren angewendet. In einem ersten Schritt wird mithilfe von Regressionsanalysen untersucht, welchen Einfluss unterschiedliche Altersgruppen in der Vergangenheit auf zentrale makroökonomische Größen hatten. Die Regressionsanalysen basieren auf den Daten der Jahre 1980 bis 2015. Um mit einer größeren Datenmenge arbeiten zu können, werden neben den sieben Ländern, für welche die Simulationen errechnet werden sollen, sechs weitere westliche Industrieländer berück- 
sichtigt (Belgien, Dänemark, Finnland, die Niederlande, Schweden, Großbritannien). Die Schätzungen verwenden jeweils verschiedene Modellspezifikationen, die auch länderspezifische Besonderheiten berücksichtigen (country fixed effects).

In einem zweiten Schritt werden die Ergebnisse der Regressionsanalysen mit Bevölkerungsprojektionen, die bis 2050 reichen, kombiniert. Für die europäischen Länder werden die Vorausberechnungen von Eurostat herangezogen, für die USA und Japan wird mit den Vorausberechnungen der United Nations, World Population Prospects 2019, gearbeitet. Diese Projektionen werden dann mit Kennzahlen des Arbeitseinsatzes aus dem Ageing Report 2018 der EU (altersspezifische Partizipationsquoten, durchschnittliche Arbeitsstunden usw.) verknüpft.

Um den Effekt einer sich ändernden Altersstruktur der Bevölkerung identifizieren zu können, sind zwei Simulationsrechnungen erforderlich. In einer ersten Simulationsrechnung wird unterstellt, dass die Bevölkerung in jedem Land auf dem Stand des Jahres 2018 „eingefroren“ wird (Basisszenario). Die Ergebnisse dieses hypothetischen Basisszenarios geben an, wie sich die Wirtschaft eines Landes bis 2050 entwickeln würde, wenn die Bevölkerungszahl und ihre Struktur konstant bleiben, aber die restlichen exogenen Erklärungsfaktoren in den Regressionsmodellen sich entsprechend den Annahmen ändern. In einer zweiten Simulationsrechnung wird anstelle der konstanten Bevölkerung des Jahres 2018 mit den genannten Bevölkerungsprojektionen gearbeitet. Die Differenz der beiden Simulationsresultate kann als demografiebedingter ökonomischer Effekt interpretiert werden wie Abbildung 1 am Beispiel der gesamtwirtschaftlichen Sparquote in Deutschland verdeutlicht. Während die gesamtwirtschaftliche Sparquote im Basisszenario bis 2050 nahezu konstant bleibt, werden im Zuge des demografischen Wandels der starke Anstieg der Personen im Rentenalter und die gleichzeitige Schrumpfung der Erwerbsbevölkerung dazu führen, dass die gesamtwirtschaftliche Sparquote in den kommenden Dekaden deutlich sinkt (zwischen 2018 und 2040 bzw. 2050 um fast 3 Prozentpunkte).

\section{Ergebnisse der Simulationsberechnungen für Deutschland, Japan und die USA}

Ausgangspunkt der nachfolgenden Simulationsberechnungen sind die Bevölkerungsprognosen bis 2050. Aus Gründen der Übersichtlichkeit beschränken sich die Ergebnisse auf Deutschland, Japan und die USA. Japan und die USA weisen von allen sieben Ländern, für die diese Berechnungen durchgeführt werden, die beiden extremsten Bevölkerungsentwicklungen auf: Japan ist das Land mit der stärksten Bevölkerungsalterung bis 2050, in den USA altert die Bevölkerung hingegen am wenigsten. Dies zeigt sich unter anderem an der Entwicklung der Bevölkerung im erwerbsfähigen Alter, welche die Menschen im Alter von 15 bis 64 Jahren umfasst (vgl. Abbildung 2).

Eine gesellschaftliche Alterung, die sich auch auf Erwerbstätige bezieht, dämpft das Wachstum der Produktivität je geleisteter Arbeitsstunde. In Japan ist die Produktivität je Arbeitsstunde 2050 alterungsbedingt 5,50 Euro geringer als im Basisszenario ohne den demografischen Wandel. Dies entspricht rund $8 \%$ des Produktivitätswerts im Basisszenario (vgl. Tabelle 1). In Deutschland liegt die Produktivität 2050 alterungsbedingt 1,90 Euro bzw. 2,3\% unter dem Wert mit einer unveränderten Bevölkerungsstruktur. Die USA können wegen der - aus ökonomischer Sicht - positiven Entwicklung ihrer Erwerbsbevölkerung 2050 hingegen einen demografiebedingten Zuwachs der Arbeitsproduktivität um rund 1,10 Euro je Stunde bzw. $1,5 \%$ erwarten.

In Deutschland und Japan bewirkt der demografisch bedingte schwächere Anstieg der Arbeitsproduktivität in Kombination mit einer wachsenden Zahl von Menschen im Rentenalter in Relation zur Zahl der Menschen im erwerbsfähigen Alter eine spürbare Dämpfung des BIP-proKopf-Wachstums. 2050 ist das für Deutschland berechnete reale BIP je Einwohner*in demografiebedingt rund 6.050 Euro geringer als im Basisszenario (vgl. Tabelle 2). Das entspricht $10,6 \%$ des BIP-pro-Kopf im Fall einer konstanten Bevölkerung. In Japan fällt die Dämpfung des BIP-pro-Kopf mit rund 12.500 Euro (19,2\% des ProKopf-Einkommens des Basisszenarios) noch größer aus. Selbst in den USA bewirkt der Anstieg des zahlenmäßigen Verhältnisses von Menschen im Rentenalter zu den Personen im erwerbsfähigen Alter ein geringeres reales BIP je Einwohner*in. Die Differenz zum Basisszenario fällt mit rund 2.300 Euro bzw. 3,8\% jedoch relativ gering aus.

Die Sparquote ist in allen drei Ländern aufgrund des steigenden Altenquotienten im Alternativszenario geringer als im Basisszenario (vgl. Tabelle 3). Die demografiebedingte Änderung der Investitionsquote fällt in den drei Ländern unterschiedlich aus. Der mit der gesellschaftlichen Alterung einhergehende erwartete Rückgang dieser Quote ist in Japan am deutlichsten ausgeprägt (vgl. Tabelle 4). In den USA fällt die Investitionsquote 2050 demografiebedingt höher aus als in dem hypothetischen Szenario mit einer konstanten Bevölkerung. Verantwortlich dafür ist der Anstieg der Erwerbsbevölkerung, für die Arbeitsplätze geschaffen werden müssen, was wiederum entsprechende Investitionen erfordert.

In Deutschland gibt es 2050 nahezu keinen Unterschied zwischen den Investitionsquoten in beiden Szenarien. 


\section{Abbildung 1}

Entwicklung der gesamtwirtschaftlichen Sparquote in Deutschland
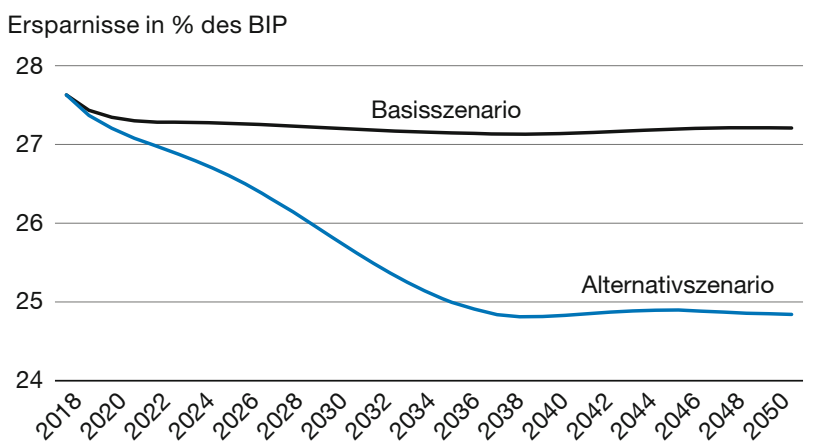

Quelle: Bertelsmann Stiftung (2019), Detailauswertung.

Verantwortlich für dieses auf den ersten Blick überraschende Ergebnis ist vor allem der Umstand, dass der Schrumpfungsprozess der erwerbsfähigen Bevölkerung zwischen 2040 und 2050 zwischenzeitlich zum Erliegen kommt, wie Abbildung 2 zu entnehmen ist, und damit ein negativer Effekt auf die Investitionen wegfällt.

Die demografisch bedingte Entwicklung des Leistungsbilanzsaldos (in Relation zum BIP) entspricht für Deutschland dem theoretisch erwarteten Verlauf: Die sinkende Sparquote ist mit steigendem Konsum im Inland verbunden. Dadurch stehen weniger Güter und Dienstleistungen für den Export zur Verfügung und gleichzeitig wird mehr importiert. Das steigende Preisniveau hat zur Folge, dass die Nachfrage aus dem Ausland nach deutschen Produkten tendenziell zurückgeht. Alterungsbedingt ist der für 2050 berechnete Leistungsbilanzüberschuss 2,3 Prozentpunkte kleiner als im Basisszenario (vgl. Tabelle 5). Die japanische Leistungsbilanz weist bereits jetzt ein Defizit auf. Alterungsbedingt ist mit einem etwas geringeren Defizit zu rechnen.

Dieses überraschende Ergebnis lässt sich wie folgt erklären: Der Leistungsbilanzsaldo ist die Differenz zwischen den gesamtwirtschaftlichen Ersparnissen und Investitionen. Wenn altersstrukturbedingt die Investitionsquote stärker sinkt als die Sparquote, wird das Leistungsbilanzdefizit kleiner. Dies ist in Japan der Fall: Dort liegt die Investitionsquote 2050 rund 4,5 Prozentpunkte unter der Quote des Basisszenarios (vgl. Tabelle 4), bei der Sparquote beträgt der Unterschied nur 3,5 Prozentpunkte (vgl. Tabelle 3). Für die USA ist wiederum ein Anstieg des bereits bestehenden Leistungsbilanzdefizits zu erwarten. Grund dafür ist vor allem der Umstand, dass ein alterungsbedingter Anstieg der Konsumquote auf eine demografisch bedingte Steigerung der Investitionsquo-

\section{Abbildung 2}

Entwicklung der erwerbsfähigen Bevölkerung

Bevölkerung im erwerbsfähigen Alter (15 bis 64 Jahre)

Indexwert, $2018=100$

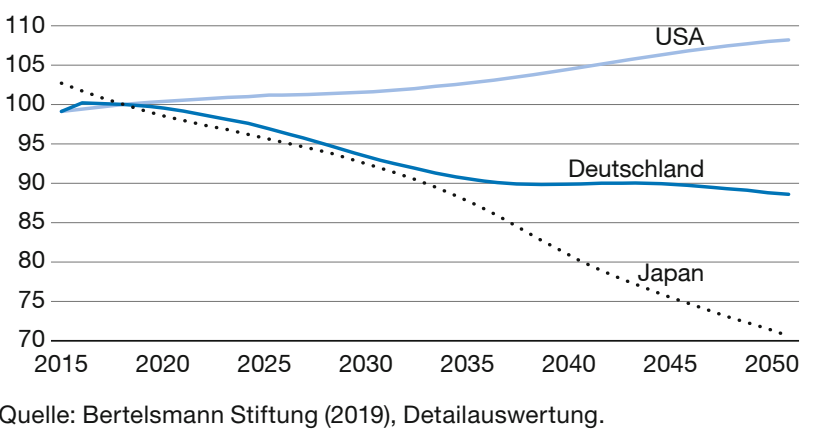

te trifft. Beides führt zu einer höheren Binnennachfrage, was das Leistungsbilanzdefizit erhöht.

\section{Demografieinduzierter technologischer Fortschritt}

Abschließend soll etwas ausführlicher auf die Bedeutung der demografischen Entwicklung für den technologischen Fortschritt eingegangen werden. Ausgangspunkt ist dabei die Überlegung, dass der demografisch bedingte Arbeitskräftemangel zu einem Lohnanstieg führt. Von Unternehmen ist zu erwarten, dass sie den teurer gewordenen Produktionsfaktor Arbeit durch Kapital ersetzen und die Arbeitsplätze dadurch kapitalintensiver werden. Die Produktionstechnologie würde dabei unverändert bleiben. Eine weitere erwartbare Reaktion von Unternehmen auf einen Lohnanstieg ist eine Erhöhung der Forschungsund Entwicklungsausgaben, um einen arbeitssparenden technologischen Fortschritt zu forcieren. Dies würde bedeuten, dass die Produktionstechnologien durch Automatisierung und Digitalisierung dahingehend verändert werden, dass sie fehlende Arbeitskräfte kompensieren. Beide Formen des Kapitaleinsatzes wirken sich wiederum positiv auf das Wachstum der Totalen Faktorproduktivität (TFP) und auf die Arbeitsproduktivität aus. Die TFP gilt dabei als Indikator für den technologischen Fortschritt einer Volkswirtschaft. Da der technologische Fortschritt jedoch nicht direkt beobachtbar ist, wird der Teil des wirtschaftlichen Wachstums, der nicht auf eine Veränderung der Quantität und Qualität der beiden Produktionsfaktoren Arbeit und Kapital zurückgeführt werden kann, gemeinhin als ein Ausdruck des technologischen Fortschritts und der Effizienzsteigerung angesehen.

Dieser Einfluss der demografischen Entwicklung auf den technologischen Fortschritt ist in der jüngsten Vergangenheit von mehreren Ökonom*innen untersucht worden. 
Tabelle 1

Produktivität je Arbeitsstunde

in Euro zu Preisen von 2010

\begin{tabular}{lcccc} 
& 2020 & 2030 & 2040 & 2050 \\
\hline Deutschland & & & & \\
\hline Basisszenario & 49,4 & 58,4 & 68,2 & 79,5 \\
\hline Alternativszenario & 49,4 & 57,6 & 67,1 & 77,6 \\
\hline Japan & & & & \\
\hline Basisszenario & 40,4 & 47,9 & 57,5 & 68,4 \\
\hline Alternativszenario & 40,4 & 47,3 & 54,2 & 62,8 \\
\hline USA & & & & \\
\hline Basisszenario & 49,4 & 56,4 & 63,4 & 71,3 \\
\hline Alternativszenario & 49,2 & 56,4 & 64,2 & 72,4 \\
\hline
\end{tabular}

Quelle: Bertelsmann Stiftung (2019), Detailauswertung.

Tabelle 3

Gesamtwirtschaftliche Sparquote

Ersparnisse in \% des BIP

$2020 \quad 2030 \quad 2040 \quad 2050$

\begin{tabular}{lcccc}
\hline Deutschland & 27,3 & 27,2 & 27,1 & 27,2 \\
\hline Basisszenario & 27,2 & 25,8 & 24,8 & 24,8 \\
\hline Alternativszenario & & & & \\
\hline Japan & 24,8 & 25,1 & 25,3 & 25,2 \\
\hline Basisszenario & 24,5 & 24,0 & 22,7 & 21,7 \\
\hline Alternativszenario & & & & \\
\hline USA & 17,0 & 17,3 & 17,5 & 17,6 \\
\hline Basisszenario & 16,8 & 15,8 & 15,5 & 15,4 \\
\hline Alternativszenario & & & &
\end{tabular}

Quelle: Bertelsmann Stiftung (2019), Detailauswertung.

Tabelle 5

Leistungsbilanzsaldo

Leistungsbilanzsaldo in \% des BIP

\begin{tabular}{lcccc}
\hline Deutschland & 2020 & 2030 & 2040 & 2050 \\
\hline Basisszenario & 6,6 & 5,8 & 5,6 & 5,7 \\
\hline Alternativszenario & 6,5 & 5,1 & 4,8 & 3,4 \\
\hline Japan & & & & \\
\hline Basisszenario & $-0,3$ & $-1,8$ & $-1,2$ & $-1,0$ \\
\hline Alternativszenario & 0,2 & $-0,4$ & $-0,7$ & 0,1 \\
\hline USA & & & & \\
\hline Basisszenario & $-4,0$ & $-1,9$ & $-1,8$ & $-1,9$ \\
\hline Alternativszenario & $-4,6$ & $-3,9$ & $-4,3$ & $-4,8$ \\
\hline
\end{tabular}

Quelle: Bertelsmann Stiftung (2019), Detailauswertung.

So haben beispielsweise Acemoglu und Restrepo (2017, 177) gezeigt, dass Länder mit einer rasch alternden Arbeitsbevölkerung in größerem Ausmaß Roboter in der Produktion einsetzen als Länder, in denen sich die Alterung der Erwerbstätigen langsamer entwickelt.
Tabelle 2

Reales Bruttoinlandsprodukt je Einwohner*in in Euro zu Preisen von 2010

\begin{tabular}{lcccc} 
& 2020 & 2030 & 2040 & 2050 \\
\hline Deutschland & & & & \\
\hline Basisszenario & 36.614 & 42.184 & 49.224 & 57.354 \\
\hline Alternativszenario & 36.393 & 40.572 & 45.508 & 51.299 \\
\hline Japan & & & & \\
\hline Basisszenario & 37.257 & 45.234 & 54.525 & 64.825 \\
\hline Alternativszenario & 36.878 & 42.396 & 48.058 & 52.356 \\
\hline USA & & & & \\
\hline Basisszenario & 42.348 & 48.272 & 54.030 & 60.584 \\
\hline Alternativszenario & 42.232 & 47.148 & 51.834 & 58.274 \\
\hline
\end{tabular}

Quelle: Bertelsmann Stiftung (2019), Detailauswertung.

Tabelle 4

Gesamtwirtschaftliche Investitionsquote Investitionen in \% des BIP

\begin{tabular}{lcccc} 
& 2020 & 2030 & 2040 & 2050 \\
\hline Deutschland & & & & \\
\hline Basisszenario & 20,7 & 21,4 & 21,5 & 21,5 \\
\hline Alternativszenario & 20,7 & 20,6 & 20,1 & 21,4 \\
\hline Japan & & & & \\
\hline Basisszenario & 25,1 & 26,9 & 26,5 & 26,2 \\
\hline Alternativszenario & 24,3 & 24,4 & 23,4 & 21,7 \\
\hline USA & & & & \\
\hline Basisszenario & 21,0 & 19,3 & 19,3 & 19,5 \\
\hline Alternativszenario & 21,3 & 19,7 & 19,8 & 20,2 \\
\hline
\end{tabular}

Quelle: Bertelsmann Stiftung (2019), Detailauswertung.

Die Auswirkung des demografischen Wandels auf den technologischen Fortschritt wurde in den Regressionsanalysen der hier betrachteten Studie ebenfalls eingeschätzt und anschließend in einem weiteren Szenario berücksichtigt. Neben der erwarteten demografischen Veränderung beeinflussen in diesem Modell der Konjunkturzyklus und die Preisentwicklung für Informations- und Kommunikationskapital das Investitionsverhalten. Die aus der Vergangenheit abgeleiteten Schätzungen des Einflusses der Bevölkerungsentwicklung auf Investitionen in Informations- und Kommunikationstechnologie sowie Software (IKT) fallen eher verhalten aus. Dafür gibt es zwei Ursachen:

- Erstens waren die Investitionsentscheidungen der Unternehmen in den Jahren zwischen 1980 und 2015 vorwiegend an den technologischen Möglichkeiten zur Optimierung von Produktionsabläufen und an der Entwicklung neuer Produkte und Dienstleistungen ausgerichtet. 
- Zweitens war die erwartete demografische Alterung in den meisten Ländern noch eher moderat bzw. die Bevölkerung im erwerbsfähigen Alter nahm zu.

Für die Simulation bis 2050 kommt hinzu, dass die erwartete Schrumpfung der erwerbsfähigen Bevölkerung z. B. in Deutschland zwischen 2040 und 2050 vorübergehend zum Erliegen kommt und damit zwischenzeitlich auch der Anreiz für Unternehmen nachlässt, mehr in IKT zu investieren. Da das Modell für den Anteil des IKT-Kapitals am Gesamtkapital auf die erwartete Veränderung der erwerbsfähigen Bevölkerung in den nachfolgenden zehn Jahren reagiert, sinkt bei einem erwarteten Wachstum der erwerbsfähigen Bevölkerung ceteris paribus der IKT-Anteil und damit auch das TFPWachstum, während er bei einer erwarteten Schrumpfung steigt. Je stärker die erwartete Schrumpfung ausfällt, desto stärker wächst auch die IKT-Intensität am Gesamtkapital. In Zukunft wird es dementsprechend voraussichtlich Phasen mit demografisch bedingt stärkeren und weniger starken Anreizen bzw. Investitionen in den technologischen Fortschritt und damit mit stärkeren und weniger starken positiven Rückwirkungen auf das TFP-Wachstum geben.

\section{Wirtschaftspolitische Handlungsalternativen}

Wenn eine Gesellschaft der alterungsbedingten Dämpfung des Wachstums des realen BIP je Einwohner*in entgegenwirken möchte, bieten sich dazu verschiedene Handlungsoptionen an:

- Denkbar ist eine Steigerung der Arbeitsmarktpartizipationsraten, vor allem von Frauen, Geringqualifizierten, bereits im Inland lebenden Migrant*innen und älteren Erwerbstätigen. Dadurch lässt sich das zahlenmäßige Verhältnis von erwerbstätigen Personen zu Rentnern und Pensionären erhöhen. Die Folge ist ein Anstieg des realen BIP je Kopf. Ein ähnlicher Effekt kann von einer Steigerung der Zuwanderung von jungen Erwerbstätigen erwartet werden.
- Eine andere Stellschraube wäre die Erhöhung der Arbeitsproduktivität durch eine Intensivierung und Verbesserung der Aus- und Weiterbildung oder eine höhere Kapitalintensität der Produktion. Letzteres bedeutet, dass in der Produktion mehr Maschinen, Computer, Roboter etc. eingesetzt werden. Dies erhöht die Produktivität der Erwerbstätigen. Der gleiche Effekt lässt sich erreichen, wenn der technologische Fortschritt forciert wird.

- Der alterungsbedingte Rückgang der Produktivität kann grundsätzlich durch höhere Automatisierung und Digitalisierung kompensiert werden. Die Regressionsanalysen bestätigen sogar einen stark positiven Effekt der IKT-Intensität auf das Produktivitätswachstum (Vu et al., 2020).

- Verharren die Investitionen in den technologischen Fortschritt allerdings auf dem Niveau der Vergangenheit bzw. reagiert das Investitionsverhalten weiterhin kaum auf die erwartete Alterung, sind auch langfristig keine positiven Effekte über diesen Wirkungskanal zu erwarten.

\section{Literatur}

Acemoglu, D. und P. Restrepo (2017), Secular Stagnation? The Effect of Aging on Economic Growth in the Age of Automation, American Economic Review, 107(5), 174-179.

Ademmer, M. et al. (2017), Produktivität in Deutschland - Messbarkeit und Entwicklung, Kieler Beiträge zur Wirtschaftspolitik, 12, 16.

Bertelsmann Stiftung (Hrsg.) (2019), Macroeconomic Consequences of Ageing and Directed Technological Change.

European Commission (2017), The 2018 Ageing Report. Underlying Assumptions \& Projection Methodologies, Institutional Paper, 065.

Juselius, M. und E. Takáts (2018), The enduring link between demography and inflation, BIS Working Paper, 722.

Skirbekk, V. (2004), Age and Individual Productivity: A Literature Survey, in G. Feichtinger (Hrsg.): Vienna Yearbook of Population Research, 133153.

Vu, K., P. Hanafizadeh und E. Bohlin (2020), ICT as a Driver of Economic Growth: A Survey of the Literature and Directions for Future Research, Telecommunications Policy, 44(2), 1-20.

Title: Macroeconomic Consequences of Demographic Ageing: Simulations Until 2050

Abstract: The change in the age structure of a society has farreaching consequences for the macroeconomic development of an economy. One of the expected consequences of an ageing population is that labour productivity and real GDP per capita will decline. This article presents results from combining the European Commission's approach to longterm forecasting with regression analyses covering the relation between macroeconomic key variables and indicators of demographic change. The simulation results for Germany, Japan and the USA through 2050 show how key macroeconomic indicators (labour productivity, GDP per capita, savings rate, investment rate, inflation rate and current account balance) change due to ageing. Countries with rapidly aging populations such as Japan and Germany in particular should expect a noticeable slowdown in the growth of real GDP per capita.

JEL Classification: J1, J11, C53 